IP-ASTP-07

CU-TP-701

SNUTP-95-067

\title{
The Chern-Simons Coefficient in Supersymmetric Yang-Mills Chern-Simons Theories
}

\author{
Hsien-Chung Kao \\ Institute of Physics, Academia Sinica \\ Nankang, Taipei, 11529 Taiwan \\ Kimyeong Lee \\ Physics Department, Columbia University \\ New York, N.Y. 10027, U.S.A. \\ and \\ Taejin Lee \\ Physics Department, Kangwon National University \\ Chuncheon, 200-701, Korea
}

We study one-loop correction to the Chern-Simons coefficient $\kappa=k / 4 \pi$ in $N=$ 1, 2, 3 supersymmetric Yang-Mills Chern-Simons systems. In the pure bosonic case, the shift of the parameter $k$ is known to be $k \rightarrow k+c_{v}$, where $c_{v}$ is the quadratic Casimir of the gauge group. In the $N=1$ case, the fermionic contribution cancels the bosonic contribution by half and the shift is $k \rightarrow k+c_{v} / 2$, making the theory anomalous if $c_{v}$ is odd. In the $N=2,3$ cases, the fermionic contribution cancels the bosonic contribution completely and there is no correction. We also calculate the mass corrections, showing the supersymmetry is preserved. As the matter fields decouple from the gauge field in the pure Chern-Simons limit, this work sheds some 
light on the regularization dependency of the correction in pure Chern-Simons systems. We also discuss the implication to the case when the gauge symmetry is spontaneously broken by the Higgs mechanism. 
There has been recently some interest in the regularization dependency of the quantum correction to the Chern-Simons coefficient. For the quantum theory to be invariant under the large gauge transformations, the Chern-Simons coefficient $k$ should be an integer[1]. In the Yang-Mills Chern-Simons theories, it is known that due to quantum correction the coefficient is shifted from $k \rightarrow k+c_{v}[2,3]$. If we take a limit where the Yang-Mills term disappear, we could conclude that there is a nonzero correction even in the pure Chern-Simons theory. However, the naive calculation in the pure Chern-Simons theory does not show such a correction[4]. Also we can use a somewhat complicated regularization to get many different corrections to the coefficient[5]. In short, there seems to be no clear principle to determine the quantum correction to the Chern-Simons coefficient in the pure Chern-Simons theory.

In this paper we study the $N=1,2,3$ supersymmetric Yang-Mills ChernSimons systems. It is known that the maximal supersymmetry is $N=3$ as the massive vector multiplet can carry spin $1,1 / 2,0,-1 / 2$ up to the sign[6]. As the Chern-Simons coefficient also appears in the mass term for the matter fields, the supersymmetry puts an additional constraint on the quantum correction to the parameter $\kappa$. Since one-loop corrections in the supersymmetric theories turn out to be finite without any regularization, there is no regularization dependency in the quantum correction. Interestingly, the matter part of the supermultiplet decouples the gauge field in the pure Chern-Simons limit. Thus, the correction in the pure Chern-Simons theory depends on whether there are other matter fields even though there is no interaction between them. Of course this is another disguise of the regularization dependency. However, we have an additional structure, supersymmetry, in the theory which we should respect, and so obtain a unique correction for each given supersymmetry. We should emphasize the correction to the Chern-Simons coefficient is meaningful only if there is any physical process which explores the physics of distance shorter than the Compton wavelength of the gauge bosons.

One-loop corrections, especially due to tadpole diagrams, can be linearly divergent and disappear in the dimensional regularization. In our supersymmetric models the linear divergences explicitly cancel each other, making one-loop corrections finite even before the regularization. While the superfield formalism is more 
efficient, here we choose to calculate the one-loop corrections in the components to compare it with the known result by Pisarski and Rao for the bosonic part [2]. Their work was done in Euclidean time with the dimensional regularization and our work is done in Minkowski time. Our results agree with theirs where they overlap.

The plan of this paper is as follows. We introduce the $N=3$ supersymmetric Lagrangian and its supersymmetric transformations. Straightforward truncations lead to the $N=1,2$ models. Then we study one-loop corrections in general and establish the method to calculate the renormalized coupling constants and masses. After that we perform the relevant one-loop calculations and present the renormalized parameters. Finally we discuss the implications of our results to the pure Chern-Simons theories and to the quantum correction in the broken phases of the self-dual Chern-Simons Higgs theories.

First, we present the $N=3$ supersymmetric Yang-Mills Chern-Simons theory of a given gauge group with an explicit $O(3)$ symmetry. The gauge multiplet is made of the field $A_{\mu}$ for one massive vector of spin 1 , and the field $\lambda_{a}$ for three Majorana fermions of spin $1 / 2$, and the field $C_{a}$ for three neutral scalar bosons and the field $\chi$ for a Majorana fermion of spin $-1 / 2$. The super Yang-Mills Lagrangian can be obtained from the dimensional reduction of the pure $N=2$ super Yang-Mills theory in four dimensions,

$$
\begin{aligned}
\mathcal{L}_{Y M}=\frac{1}{g^{2}} \operatorname{tr}\{ & -\frac{1}{2} F_{\mu \nu}^{2}+\left(D_{\mu} C_{a}\right)^{2}+\left(D_{a}\right)^{2}+i \bar{\lambda}_{a} \not D_{\mu} \lambda_{a}+i \bar{\chi} \not_{\mu} \chi \\
& \left.+i \epsilon_{a b c} \bar{\lambda}_{a}\left[\lambda_{b}, C_{c}\right]-2 i \bar{\lambda}_{a}\left[\chi, C_{a}\right]-\frac{1}{2}\left[C_{a}, C_{b}\right]\left[C_{b}, C_{a}\right]\right\}
\end{aligned}
$$

where $D_{\mu}=\partial_{\mu}-i\left[A_{\mu},\right]$, and $a, b, c=1,2,3$. The gauge group generators in the fundamental representation satisfy $\left[T^{m}, T^{n}\right]=i f^{l m n} T^{l}$, and $\operatorname{tr} T^{m} T^{n}=\delta^{m n} / 2$. The fields belong to the adjoint representation and $A_{\mu}=A_{\mu}^{m} T^{a}$ et. cetra. The quadratic Casimir number $c_{v}$ for the group is given by $f^{k m n} f^{l m n}=c_{v} \delta^{k l}$. Here, the metric is given by $\eta_{\mu \nu}=\operatorname{diag}(1,-1,-1)$ and $\epsilon^{012}=\epsilon_{012}=1$. The pure imaginary gamma matrices satisfy $\gamma^{\mu} \gamma^{\nu}=\eta^{\mu \nu}-i \epsilon^{\mu \nu \rho} \gamma_{\rho}$. The dimensional reduction of the $N=2$ supersymmetry implies that this theory has the not-so-obvious $N=4$ supersymmetry. 
By supersymmetrizing the Chern-Simons term, we get the supersymmetric Chern-Simons Lagrangian[6],

$\mathcal{L}_{C S}=\kappa \operatorname{tr}\left\{\epsilon^{\mu \nu \rho}\left(A_{\mu} \partial_{\nu} A_{\rho}-\frac{2}{3} i A_{\mu} A_{\nu} A_{\rho}\right)-\bar{\lambda}_{a} \lambda_{a}+\bar{\chi} \chi+2 C_{a} D_{a}+\frac{i}{3} \epsilon_{a b c} C_{a}\left[C_{b}, C_{c}\right]\right\}$.

The Lagrangian we study is the sum of $\mathcal{L}_{Y M}$ and $\mathcal{L}_{C S}[6]$. Note that the corresponding $N=2$ theories can be easily obtained by setting $C_{1}=C_{2}=D_{1}=D_{2}=$ $\lambda_{3}=\chi=0$. The $N=1$ theories are obtained from $N=2$ theories by further imposing $C_{3}=0, \lambda_{2}=0$. The $N=3$ supersymmetric transformation of the fields is given by

$$
\begin{aligned}
\delta A_{\mu}= & -i \bar{\alpha}_{a} \gamma_{\mu} \lambda_{a}, \\
\delta \lambda_{a}= & i \not B \alpha_{a}-\epsilon_{a b c}\left(D_{b} \alpha_{c}-i \not D C_{b} \alpha_{c}\right)+i\left[C_{a}, C_{b}\right] \alpha_{b}, \\
\delta \chi= & -i \not D C_{a} \alpha_{a}-D_{a} \alpha_{a}+\frac{i}{2} \epsilon_{a b c}\left[C_{b}, C_{c}\right] \alpha_{a}, \\
\delta C_{a}= & -\epsilon_{a b c} \bar{\alpha}_{b} \lambda_{c}+\bar{\alpha}_{a} \chi, \\
\delta D_{a}= & i \epsilon_{a b c} \bar{\alpha}_{b} \not D \lambda_{c}+i \bar{\alpha}_{a} \not D \chi+i\left[\bar{\alpha}_{b} \lambda_{a}, C_{b}\right] \\
& \quad-i\left[\bar{\alpha}_{b} \lambda_{b}, C_{a}\right]+i\left[\bar{\alpha}_{a} \lambda_{b}, C_{b}\right]-i \epsilon_{a b c} \bar{\alpha}_{b}\left[\chi, C_{c}\right],
\end{aligned}
$$

where $B^{\mu}=\epsilon^{\mu \nu \rho} \partial_{\nu} A_{\rho}$. While we do not know whether there is a $N=3$ superfield formalism, clearly it can be written in terms of the $N=1$ or $N=2$ superfields[7].

By using the field equation $D_{a}+\kappa g^{2} C_{a}=0$, of the Lagrangian $\mathcal{L}_{Y M}+\mathcal{L}_{C S}$, we can eliminate the auxiliary field $D_{a}$ and obtain the following on-shell Lagrangian:

$$
\begin{aligned}
& \mathcal{L}=\frac{1}{g^{2}} \operatorname{tr}\{-\frac{1}{2} F_{\mu \nu}^{2}+\left(D_{\mu} C_{a}\right)^{2}+i \bar{\lambda}_{a} \gamma^{\mu} D_{\mu} \lambda_{a}+i \bar{\chi} \gamma^{\mu} D_{\mu} \chi \\
&\left.+i \epsilon_{a b c} \bar{\lambda}_{a}\left[\lambda_{b}, C_{c}\right]-2 i \bar{\lambda}_{a}\left[\chi, C_{a}\right]-\frac{1}{2}\left[C_{a}, C_{b}\right]\left[C_{b}, C_{a}\right]\right\} \\
&+\kappa \operatorname{tr}\left\{\epsilon^{\mu \nu \rho}\left(A_{\mu} \partial_{\nu} A_{\rho}-\frac{2}{3} i A_{\mu} A_{\nu} A_{\rho}\right)-\kappa g^{2} C_{a}^{2}-\bar{\lambda}_{a} \lambda_{a}+\bar{\chi} \chi\right. \\
&\left.\quad-\frac{i}{3} \epsilon_{a b c} C_{a}\left[C_{b}, C_{c}\right]\right\} .
\end{aligned}
$$

Due to the mass term for $C_{a}$, the ground state of the theory is the symmetric vacuum where $\left\langle C_{a}\right\rangle_{v}=0$. This contrasts the pure Yang-Mills case whose vacua have a flat direction. 
We are here interested in calculating one-loop corrections to the theory. If we scale the gauge field by $A_{\mu}^{m} \rightarrow g A_{\mu}^{m}$, we can see the expansion parameter is $g^{2}$, which has a dimension of mass. The dimensionless coupling constant turns out to be $1 /|\kappa|$, which should be small for the perturbative expansion to work. With the usual covariant gauge fixing term,

$$
\mathcal{L}_{g f}=-\frac{1}{2 \xi}\left(\partial^{\mu} A_{\mu}^{m}\right)^{2}
$$

we get the Fadeev-Popov ghost Lagrangian,

$$
\mathcal{L}_{F P}=\partial^{\mu} \bar{\eta}^{m} \partial_{\mu} \eta^{m}+f^{l m n} \partial^{\mu} \bar{\eta}^{l} A_{\mu}^{m} \eta^{n}
$$

Combining the Lagrangian (4) and the gauge fixing terms (5) and (6), we obtain the quadratic terms

$$
\begin{aligned}
\mathcal{L}_{0} & =\frac{1}{2 g^{2}} A^{m \mu}\left\{\left(\partial^{2} \eta_{\mu \nu}-\partial_{\mu} \partial_{\nu}\right)-m \epsilon_{\mu \nu \rho} \partial^{\rho}+\frac{1}{\xi} \partial_{\mu} \partial_{\nu}\right\} A^{m \nu} \\
& +\frac{1}{2 g^{2}} C_{a}\left\{-\partial^{2}-m^{2}\right\} C_{a}+\frac{1}{2 g^{2}} \bar{\lambda}_{a}\{i \not \partial-m\} \lambda_{a}+\frac{1}{2 g^{2}} \bar{\chi}\{i \not \partial+m\} \chi \\
& +\bar{\eta}^{m}\left(-\partial^{2}\right) \eta^{m}
\end{aligned}
$$

where $m \equiv \kappa g^{2}$. From the quadratic terms (7), it is straightforward to get the propagators for the gauge, scalar, and fermion fields. Here we show only the propagator for the gauge field,

$$
\left(\Delta_{0}\right)_{\mu \nu}(p)=\frac{-i g^{2}\left(p^{2} \eta_{\mu \nu}-p_{\mu} p_{\nu}\right)+m g^{2} \epsilon_{\mu \nu \rho} p^{\rho}}{p^{2}\left(p^{2}-m^{2}\right)}-\frac{i \xi p_{\mu} p_{\nu}}{p^{4}}
$$

To avoid the infrared divergence, we use the Landau gauge $\xi=0$.

Since the theory is finite, we can calculate perturbatively the effective action in terms of the bare fields and parameters without introducing the counter terms. 
Because of the Lorentz and gauge invariance, the gluon self-energy takes the form:

$$
\pi_{\mu \nu}(p)=\left(p^{2} \eta_{\mu \nu}-p_{\mu} p_{\nu}\right) \Pi_{e}\left(p^{2}\right)-i \epsilon_{\mu \nu \rho} p^{\rho} \Pi_{o}\left(p^{2}\right)+p_{\mu} p_{\nu} \Pi_{3}\left(p^{2}\right) .
$$

The kinetic term in the effective action for the gauge boson is then

$$
i\left(\Delta_{\mu \nu}(p)\right)^{-1}=i\left(\Delta_{0 \mu \nu}(p)\right)^{-1}+\pi_{\mu \nu}(p) .
$$

With the correction $i p^{2} \tilde{\Pi}\left(p^{2}\right)$ to the ghost propagator, we get the corrected ghost kinetic term,

$$
i(\tilde{\Delta}(p))^{-1}=i\left(\tilde{\Delta}_{0}(p)\right)^{-1}+p^{2} \tilde{\Pi}(p) .
$$

The part of the effective action which is similar to the classical Lagrangian can be written in terms of the renormalized fields and parameters with the standard normalization. This leads the relation between the renormalized fields and parameters and the bare fields and parameters. For example,

$$
\begin{aligned}
A_{\mu}^{m} & =\sqrt{Z_{3}} A_{\mathrm{ren} \mu}^{m}, \\
\eta^{m} & =\sqrt{\tilde{Z}} \eta_{\mathrm{ren}}^{m} .
\end{aligned}
$$

From Eq.(11) we see the ghost field renormalization factor is

$$
\tilde{Z}=1-\tilde{\Pi}(0) .
$$

With our normalization of the gauge field kinetic term, the interaction term between the ghost fields and the vector boson should be again unity after renormalization by the Ward indentity, which means that

$$
Z_{3}=\tilde{Z}^{-2}
$$

With the definition $Z_{k} \equiv 1-\Pi_{0}(0) / \kappa$, the renormalized Chern-Simons coefficient is

$$
\begin{aligned}
\kappa_{\text {ren }} & =\kappa Z_{k} Z_{3}=\kappa Z_{k} \tilde{Z}^{-2} \\
& =\kappa\left(1-\frac{1}{\kappa} \Pi_{o}(0)+2 \tilde{\Pi}(0)\right) .
\end{aligned}
$$

The renormalized coupling $g_{\mathrm{ren}}^{2}=g^{2}\left(Z_{g} Z_{3}\right)^{-1}$ with $Z_{g}=1+g^{2} \Pi_{e}(0)$ is not much interesting. The renormalized mass for the vector bosons can be obtained 
from Eq.(9). We calculate the pole of the propagator at $p^{2}=m^{2}$ and so

$$
m_{\mathrm{ren}}=\left[1+g^{2} \Pi_{e}\left(m^{2}\right)-\frac{1}{\kappa} \Pi_{o}\left(m^{2}\right)\right] m .
$$

We can also calculate the similar mass corrections to the matter fields. As the supersymmetry is preserved, the renormalized mass for the matter fields will turn out to be identical to that of the vector bosons.

We first calculate the correction to the ghost propagator

$$
\tilde{\Pi}\left(p^{2}\right)=\frac{i c_{v} m}{\kappa p^{2}} \int \frac{d^{3} k}{(2 \pi)^{3}} \frac{\left[k^{2} p^{2}-(k \cdot p)^{2}\right]}{k^{2}\left(k^{2}-m^{2}\right)(k-p)^{2}} .
$$

There are seven Feynman graphs contributing to the gluon self-energy, but only three of them yield non-vanishing corrections to $\Pi_{o}$ : one gluon loop and two fermion loops. Because of the supersymmetry, the result is free of ultraviolet divergence and there is no need of regularization. After some algebra we have the bosonic and fermionic contributions,

$$
\begin{aligned}
& \Pi_{o}^{B}\left(p^{2}\right)=\frac{i c_{v} m}{p^{2}} \int \frac{d^{3} k}{(2 \pi)^{3}} \frac{\left[k^{2} p^{2}-(k \cdot p)^{2}\right]\left[5 k^{2}-5 k \cdot p+4 p^{2}-2 m^{2}\right]}{k^{2}\left(k^{2}-m^{2}\right)(k-p)^{2}\left[(k-p)^{2}-m^{2}\right]}, \\
& \Pi_{o}^{F}\left(p^{2}\right)=\frac{i c_{v} m}{p^{2}} \int \frac{d^{3} k}{(2 \pi)^{3}} \frac{-2 p^{2}}{\left(k^{2}-m^{2}\right)\left[(k-p)^{2}-m^{2}\right]}
\end{aligned}
$$

which we can evaluate explicitly. Since $\Pi\left(p^{2}\right)$ and $\tilde{\Pi}_{o}\left(p^{2}\right)$ vanishes at the large momentum limit, the parameter $\kappa$ does not get renormalized in short distance. The one-third of the $\lambda_{a}$ contribution is cancelled by the $\chi$ contribution. The bosonic contribution to the odd part comes only from the gauge field. We can see that both $\Pi_{o}^{B}$ and $\Pi_{o}^{F}$ are finite.

Taking the zero momentum limit, we get for the $N=3$ case that

$$
\begin{aligned}
& \tilde{\Pi}(0)=\frac{-c_{v}}{6 \pi|\kappa|}, \\
& \Pi_{o}^{B}(0)=\frac{-7 c_{v} \kappa}{12 \pi|\kappa|}, \\
& \Pi_{o}^{F}(0)=\frac{c_{v} \kappa}{4 \pi|\kappa|} .
\end{aligned}
$$

The above corrections hold equally well for the $N=2$ case. But for the $N=1$ 
case, the fermionic contribution $\Pi_{o}^{F}(0)$ should be one half of the above result. From Eq. (15), we see immediately see that

$$
\begin{aligned}
& \left.\kappa_{\text {ren }}\right|_{N=2,3}=\kappa, \\
& \left.\kappa_{\text {ren }}\right|_{N=1}=\kappa+\frac{c_{v} \kappa}{8 \pi|\kappa|},
\end{aligned}
$$

to one loop order. The shift in $k=4 \pi \kappa$ for the $N=1$ case is $c_{v} / 2$, which is a half integer if $c_{v}$ is odd. In this case, the theory would be quantum mechanically anomalous.

For the mass correction, we calculate the $\Pi_{e}\left(p^{2}\right)$ for the $N=3$ case, which is the sum of the bosonic contribution $\Pi_{e}^{B}\left(p^{2}\right)$ and the fermionic contribution $\Pi_{e}^{F}\left(p^{2}\right)$ :

$$
\begin{aligned}
& \Pi_{e}^{B}\left(p^{2}\right)=\frac{i c_{v}}{2 p^{4}} \int \frac{d^{3} k}{(2 \pi)^{3}}\left\{\frac{-6\left[k^{2} p^{2}-(k \cdot p)^{2}\right]}{\left(k^{2}-m^{2}\right)\left[(k-p)^{2}-m^{2}\right]}+\frac{6 p^{2}}{\left(k^{2}-m^{2}\right)}\right. \\
&+\frac{\left[k^{2} p^{2}-(k \cdot p)^{2}\right] Q}{k^{2}\left(k^{2}-m^{2}\right)(k-p)^{2}\left[(k-p)^{2}-m^{2}\right]} \\
&\left.+\frac{(8 / 3) p^{2}}{\left(k^{2}-m^{2}\right)}\right\}, \\
& \Pi_{e}^{F}\left(p^{2}\right)=\frac{i c_{v}}{2 p^{4}} \int \frac{d^{3} k}{(2 \pi)^{3}}\left\{\frac{-8(k \cdot p)^{2}+8(k \cdot p) p^{2}+8 m^{2} p^{2}}{\left(k^{2}-m^{2}\right)\left[(k-p)^{2}-m^{2}\right]}\right\},
\end{aligned}
$$

where $Q \equiv-3 k^{2}(k-p)^{2}-2 k^{2} p^{2}-4(k-p)^{2} p^{2}-2(k \cdot p)^{2}+m^{2} k^{2}-m^{2}(k \cdot p)$. The first line in $\Pi_{e}^{B}\left(p^{2}\right)$ comes from the scalar loop diagrams, the second line comes from the combination of the gluon and ghost loop diagrams. and the third line comes from the gluon tadpole diagram. Note that in $\Pi_{e}^{F}\left(p^{2}\right)$, the contributions from $\lambda$ and $\chi$ loops are of the same sign, unlike the case in $\Pi_{o}^{F}\left(p^{2}\right)$. The corresponding $N=2$ results can be obtained by multiplying a factor of $1 / 3$ to the scalar diagrams and a factor of $1 / 2$ to the fermion diagrams. The results for $N=1$ is obtained by dropping the scalar contribution and multiplying a factor $1 / 4$ to the fermion contribution. One can see easily that the linear divergences in $\Pi_{e}^{B}$ and $\Pi_{e}^{F}$ cancel each other for each $N$.

We can calculate explicitly $\Pi_{e}\left(p^{2}\right)$ and $\Pi_{o}\left(p^{2}\right)$ and get the results for the $N=3$ 
case,

$$
\begin{aligned}
& g^{2} \Pi_{e}\left(m^{2}\right)=\frac{g^{2} c_{v}}{64 \pi|m|}(5 \ln 3-4+i \pi) \\
& \Pi_{o}\left(m^{2}\right) / \kappa=-\frac{g^{2} c_{v}}{64 \pi|m|}(11 \ln 3+4-i \pi) .
\end{aligned}
$$

Note that the pole of the propagator is $p^{2}=m^{2}-i \epsilon$. Similar results can be obtained for $N=1,2$ cases. From Eq.(16), we get the mass corrections,

$$
\begin{aligned}
\left.\Delta m\right|_{N=3} & =\frac{c_{v} m}{4 \pi|\kappa|} \\
\left.\Delta m\right|_{N=2} & =\frac{3 c_{v} m}{8 \pi|\kappa|} \\
\left.\Delta m\right|_{N=1} & =\frac{c_{v} m}{2 \pi|\kappa|}
\end{aligned}
$$

As a check to the above results, we also calculated the mass corrections to all other particles in the supermultiplets and got the identical results. We expect the supersymmetry is preserved quantum mechanically and see that the supersymmetry is preserved as far as the one-loop mass correction is concerned.

We thus have obtained the shift of the Chern-Simons coefficient for the supersymmetric Yang-Mills Chern-Simons theories. For the $N=2,3$ cases, there is no correction and for the $N=1$ case, the shift is $k \rightarrow k+c_{v} / 2$. If the correction in the $N=1$ case is a half integer, the theory would be anomalous. The result depends crucially on the sign of the various terms. As a check, we also calculated the mass correction to charged particles and have shown that the supersymmetry is preserved.

There are many interesting implications arising from our results. It would be interesting to understand the possibly anomalous $N=1$ supersymmetric theory in terms of the effective action induced by the integrating over a massive Majorana fermion. This seems quite analogous to the global $Z_{2}$ anomaly discovered by Witten in the four dimensional gauge theory[8].

In calculating the knot invariant, Witten observed that the shift $\kappa \rightarrow \kappa+c_{v}$ occurs naturally in the pure Chern-Simons theory[9]. It would be also interesting 
to find the similar arguments for the supersymmetric knot invariants which is consistent with our results.

Finally, our work is also motivated partially by the recent controversy about the quantum corrections to the Chern-Simons coefficient when the gauge symmetry is spontaneously broken by the Higgs field[10]. The calculations done for both abelian and nonabelian gauge groups suggest that in the broken phase the quantum correction depends on whether the coefficient is for the unbroken symmetry generators or the broken symmetry generators. It seems that the correction for the unbroken generators is an integer[11] and that for the broken generators is a complicated function of the coupling constants. It has been argued that the corrections to the broken generators can be viewed as parts of a gauge invariant effective action which imitates the Chern-Simons term in the broken phase, which has been shown explicitly in abelian case[12].

However, there are good reasons for the correction to the Chern-Simons coefficient to be quantized even in broken phase[13]. Especially we have shown that this is indeed the case in a special class of theories, the abelian self-dual Chern-SimonsHiggs models[13]. We expect also that for the nonabelian self-dual Chern-SimonsHiggs systems the correction is quantized. In calculating the quantum corrections in these theories, one has to understand the role of the Yang-Mills term and the regularization dependency. This work answers this question.

\section{Acknowledgement}

The work by K.L. is supported in part by U.S. DOE, the NSF Presidential Young Investigator program and the Alfred P. Sloan Foundation. The work by T.L. is supported by Korea Science and Engineering Foundation (through the Center for Theoretical Physics, SNU) and the Basic Science Research Institute Program, Ministry of Education, Korea (No. BSRI-94-2401). 


\section{REFERENCES}

1. S. Deser, R. Jackiw and S. Templeton, Phys. Rev. Lett. 48, 975 (1982); S. Deser, R. Jackiw and S. Templeton, Ann. Phys. (N.Y.) 140, 372 (1982).

2. R.D. Pisarski and S. Rao, Phys. Rev. D32, 2081 (1985).

3. W. Chen, G.W. Semenoff and Y.-S. Wu, Mod. Phys. Lett. A 5, 1833 (1990); L. Álvarez-Gaumé, J.M.F. Labastida and A.V. Ramallo, Nucl. Phys. B334, 103 (1990); M. Asorey and F. Falceto, Phys. Lett. B241, 31 (1990); C.P. Martin, Phys. Lett. B241, 513 (1990); M.A. Shifman, Nucl. Phys. B352, 87 (1991).

4. E. Guadagnini, M. Martellini and M. Mintchev, Phys. Lett. B227, 11 (1989).

5. M. Asorey, F. Falceto, J.L. López and G. Luzón, Nucl. Phys. B429, 344 (1994).

6. H.-C. Kao and K. Lee, Phys. Rev. D46, 4691 (1992); H.-C. Kao, ibid. 50, 2881 (1994).

7. S.J. Gates, M.T. Grisaru, M. Rocek and W. Siegel, "Superspace or One Thousand and One Lessons in Supersymmetry," Benjamin/Cummings, Reading (1983); E.A. Ivanov, Phys. Lett. B268, 203 (1991); S.J. Gates and H. Nishino, ibid. 281, 72 (1992).

8. E. Witten, Phys. Lett. B117, 324 (1982).

9. E. Witten, Comm. Math. Phys. 117, 353 (1988).

10. S. Yu. Khlebnikov and M. Shaposhinikov, Phys. Lett. B254, 148 (1991).

11. L. Chen, G. Dunne, K. Haller and E. Lim-Lombridas, Phys. Lett. B348, 468 (1995).

12. A. Khare, R. MacKenzie and M. Paranjape, Phys. Lett. B343, 239 (1995).

13. H-C. Kao, K. Lee, C. Lee and T. Lee, Phys. Lett. B341, 181 (1994). 